\title{
Relevance of nutritional support and early rehabilitation in hospitalized patients with COPD
}

\author{
Ghislaine Gayan-Ramirez \\ Laboratory of Respiratory Diseases, Department of Chronic Diseases, Metabolism and Ageing (CHROMETA), KU Leuven, Leuven, Belgium \\ Correspondence to: Ghislaine Gayan-Ramirez. Laboratory of Respiratory Diseases, Department of Chronic Diseases, Metabolism and Ageing \\ (CHROMETA), Onderwijs en Navorsing I, Herestraat 49 bus 706, 3000 Leuven, Belgium. Email: ghislaine.gayan-ramirez@kuleuven.be.
}

\begin{abstract}
Exacerbation of COPD is associated with a worsening of lung function and symptoms, and a serious loss in functional status and health related quality of life. It also represents the major cause for emergency hospitalization in COPD patients which places the patient at risk for developing further muscle weakness. Actually, loss of muscle function developed fast and continued to decrease over time during hospitalization and may be further compromised when disease deterioration would require the use of ventilator support. Exacerbations have long term consequences on physical performance, physical activity and health-related quality of life as recovery of skeletal muscle function is slow and incomplete and may even be compromised in case of re-exacerbation. Factors such as bed rest, reduced physical activity, hypoxia, inflammation, drug treatment and negative protein balance due to insufficient dietary intake and increased resting energy expenditure are all potential contributors to skeletal muscle weakness during exacerbation. Because low levels of physical activity and poor physical performance are predictor of hospital re-admission and are associated with lower survival in these patients, interventions to prevent the additional loss of muscle function during exacerbation or to hasten functional recovery have been recently explored. This review focuses on the knowledge regarding the potential benefit of early rehabilitation and nutritional supplementation as modifiable factors susceptible to ameliorate muscle weakness during exacerbation in hospitalized COPD patients.
\end{abstract}

Keywords: Rehabilitation; nutritional support; exacerbation; COPD; hospitalization

Submitted Mar 01, 2018. Accepted for publication Mar 20, 2018.

doi: $10.21037 /$ jtd.2018.03.167

View this article at: http://dx.doi.org/10.21037/jtd.2018.03.167

\section{Introduction}

COPD is complicated by exacerbation which represents the main reason for hospitalization. Exacerbation is described as an acute event in the natural course of the disease that is characterized by a change in baseline dyspnea, cough and/ or sputum which is beyond normal day-today variations and which necessitates a change in regular medication $(1,2)$. During acute exacerbations, weight loss and muscle wasting is accelerated especially when exacerbation requires hospitalization. In fact, patients with COPD admitted to hospital with a severe exacerbation are at risk for developing skeletal muscle weakness $(3,4)$. Actually, the loss of muscle function developed rapidly and the force of the lower limb muscles continued to decrease over time during hospitalization (3-5). This muscle weakness may be further complicated when disease deterioration would require the use of ventilator support especially invasive mechanical ventilation where prolonged bed rest and the risk to become ventilator dependent may promote muscle weakness and thus increased morbidity and mortality.

Contributing factors to skeletal muscle weakness during acute exacerbation necessitating hospitalization include (prolonged) bed rest, reduced physical activity (6), hypoxia, inflammation (7) and a negative protein balance due to insufficient dietary intake and increased resting energy 
expenditure (8). In addition, drug treatment (e.g., exposure to high dosage of glucocorticoids) $(3,9)$ may further compromise skeletal muscle function. Moreover, drug related side effects may also alter the patients' willingness to move, and thus contribute to adverse effects on physical and mental health (4). In the end, this may lead to hospital readmission (10).

Exacerbations have long term consequences as spontaneous recovery of skeletal muscle function is partial and slow with quadriceps force not being returned to baseline values 3 months after hospital discharge (3) and physical activity levels being seriously reduced one month after hospital discharge compared to stable COPD patients with similar disease severity (4). Loss of exercise capacity persists despite improvement in patient' symptoms as well as lung function (11) and this impairment is of particular concern as low exercise capacity is a predictor of re-hospitalization and lower survival in this population (12). In addition, recovery may be further compromised in case of re-exacerbation as the effects of reexacerbation will build on the incomplete functional recovery. Consequently, this will delay and impair regain of skeletal muscle function. In fact, exacerbations have been associated with reduced exercise capacity (11), further impairment of quality of life (13) and increased likelihood of becoming housebound (14).

Up to now, treatment of exacerbation has usually been directed at improving ventilatory function, and less attention has been paid regarding other systemic impacts notably skeletal muscle weakness. However, given the highly deleterious effects and consequences of exacerbation on skeletal muscle function in COPD patients, several interventions have been considered during the last decade with as goal to minimize or to prevent the additional loss of muscle function during exacerbation or to hasten functional recovery. This review will thus focus on the knowledge regarding the potential benefit of early rehabilitation and nutritional support as counter measures of muscle weakness during exacerbation. As detailed thereafter, exercise training was based on either endurance training (walking or cycling) or resistance/strength training (repetitive lifting of loads). Other strategies were also used for patients not able to actively participate to such training program. These interventions include neuromuscular electrical stimulation, use of a vibrating platform and for more disabled patients, strategies ranging from passive mobilization, deambulation to endurance training when patient status improves. For nutritional support, the interventions were based on oral supplementation of either energy rich diet or protein rich diet.

\section{Early rehabilitation}

\section{Rationale}

Because of the rapid deterioration of skeletal muscle function during exacerbation and the long-lasting consequences of exacerbation on physical performance, physical activity and health-related quality of life, particular interest in the potential of using rehabilitation intervention in the acute setting of exacerbation has been grown in the last decade. Because muscle function continues to deteriorate in the absence of rehabilitation following a COPD exacerbation $(3,4)$, it is reasonable to believe that a rehabilitation intervention delivered at the time of acute illness could constitute an essential non-pharmacological element in the treatment of acute exacerbation. Actually, such strategy might offer the possibility to minimize the impact of exacerbation on skeletal muscle facilitating thereby functional recovery after exacerbation and thus limiting the risk for subsequent hospital admission.

\section{Definition, goal and modalities}

There is actually no clear definition on the time the term "early" refers to. In the current literature, the term early rehabilitation has been used for intervention starting within the first days of hospitalization, and running in parallel with the usually required medical care but also for intervention starting either immediately after discharge or after a few days or weeks. Therefore, depending on the time at which the intervention is delivered, the goal of rehabilitation will differ being preventive when applied early during hospitalization, hastening recovery in case initiated later after discharge, or being preventive and attempting to maintain its benefits when applied during hospitalization but followed by a program implanted at discharge. Trials of rehabilitation up to now consisted in either in-patient intervention delivered for the duration of hospitalization followed or not by post-discharge intervention or outpatient intervention initiated after discharge and based on either in-hospital or home rehabilitation. Finally, various types of rehabilitation (active training, non-volitional training, combination of both) and modalities (frequency of sessions, intensity and duration) have been used also depending on whether the patients are under ventilatory support or not. Limb (either upper or lower) muscles are usually targeted by rehabilitation. In active training, the patient voluntarily contract his/her muscles. Endurance 
training (also called aerobic training) is a form of active training where several groups of muscles are trained. Walking (ground-based or on a treadmill) or biking (on a stationary cycle ergometer) are typical exercise modalities of endurance training. In resistance or strength training, another type of active training, repetitive lifting of loads are used to train local muscle groups. With non-volitional training, the patient does not contract his/her muscle voluntarily and muscle contraction is achieved either by passive mobilization of the limbs or through neuro electrical stimulation of the muscle or by placing the patient on a vibrating platform. The results of the different rehabilitation trials currently available have been detailed thereafter and are presented according to the type of intervention (active or non-volitional), the time at which the intervention was initiated, and to whether the patient was under ventilatory support or not.

\section{Early rehabilitation in bospitalized non-ventilated patients}

\section{Active training (Table 1)}

Active training implies that the patient performs exercise in an active participation while contracting his/her muscles. Both aerobic and resistance/strength training has been used as rehabilitation strategy in COPD patients with exacerbation.

\section{In-patient program only}

In the randomized control trial of Troosters et al. (16) in severe COPD patients hospitalized for an acute exacerbation, an incremental resistance training was started early after hospital admission (day 2) and consisted in daily quadriceps resistance training for the duration of hospitalization (one week). This strategy did prevent deterioration of quadriceps muscle force reported during exacerbation (16). It also improved 6-minute walking distance (6MWD) at discharge although this difference was not significant compared to patients receiving usual care. Nevertheless, improvement of $6 \mathrm{MWD}$ and quadriceps muscle force was maintained after one month. Moreover, pre to post changes in quadriceps force correlated with the global muscle anabolic/catabolic index ratio. Whether this was associated with improvement in physical activity level was not assessed in the current study but at least this study supports the idea that beyond the usual medical care, early in-hospital rehabilitation during an acute exacerbation can prevent deterioration of muscle function and thus can help the patients in maintaining a certain level of function after hospitalization. Finally, this intervention seems to be safe as no adverse effects were noticed but seems also feasible even in an acute hospital setting and within 2 days of admission since $85 \%$ of the patients completed the rehabilitation course (16). This is actually the only study evaluating the effects of an exercise training program initiated within 2 days after hospital admission with a follow-up after one month.

Effectiveness and safety of a pulmonary rehabilitation program initiated on the second day of admission until discharge was further underlined in the study of $\mathrm{He}$ et al. (19). This extensive pulmonary rehabilitation program consisted in 30 min session twice daily of upper and lower limb endurance training, strength training in addition to breathing retraining and education (19). At discharge, exercise capacity and quality of life was improved in the rehabilitation group as shown by enhanced 6MWD and CRQ-SAS score and lower CAT score.

Along the same line, the use of an in-hospital whole body resistance training program for upper and lower limbs started on the third day of hospitalization for the duration of hospital stay improved upper and lower limb muscle strength at discharge, an effect that was still present one month after discharge (18). This intervention did also enhance exercise capacity (assessed by 6MWD) and health related quality of life without affecting inflammatory markers. This strategy had, however, no impact on physical activity in daily life. Adherence to resistive training was $95 \%$ with some patients performing exercises with non-invasive ventilation in $35 \%$ of the sessions because of dyspnea. This study corroborates the potential benefit, feasibility and safety of early rehabilitation for hospitalized COPD patients with acute exacerbation.

It is still unknown at which time the rehabilitation program should be initiated after admission and whether this would impact on the outcomes. Actually, a nationwide retrospective cohort study in Japan comparing early (within $48 \mathrm{~h}$ of admission) and delayed (after $48 \mathrm{~h}$ of admission) pulmonary rehabilitation reported a lower proportion of 90-day readmission and a shorter length of stay in patients with exacerbation of COPD who followed the early pulmonary rehabilitation (21). This was, however, not associated with differences in activity of daily living status at discharge based on the Barthel index. This study seems to suggest that early rehabilitation might be preferable for some outcomes but further investigation is obviously warranted to confirm these data. Indeed, it might also be argued that it would be preferable to wait until the patient has improved from this acute event before starting 


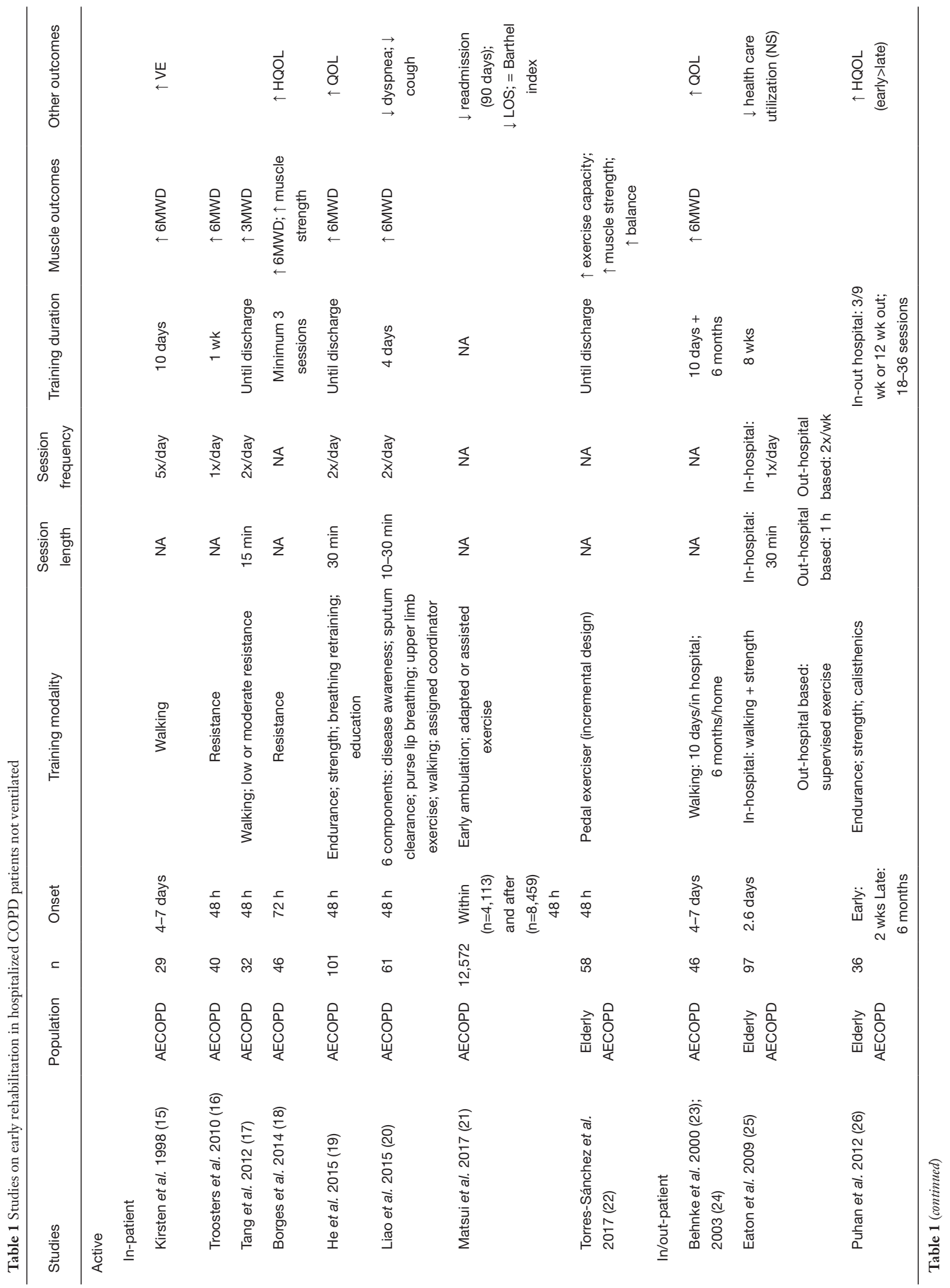




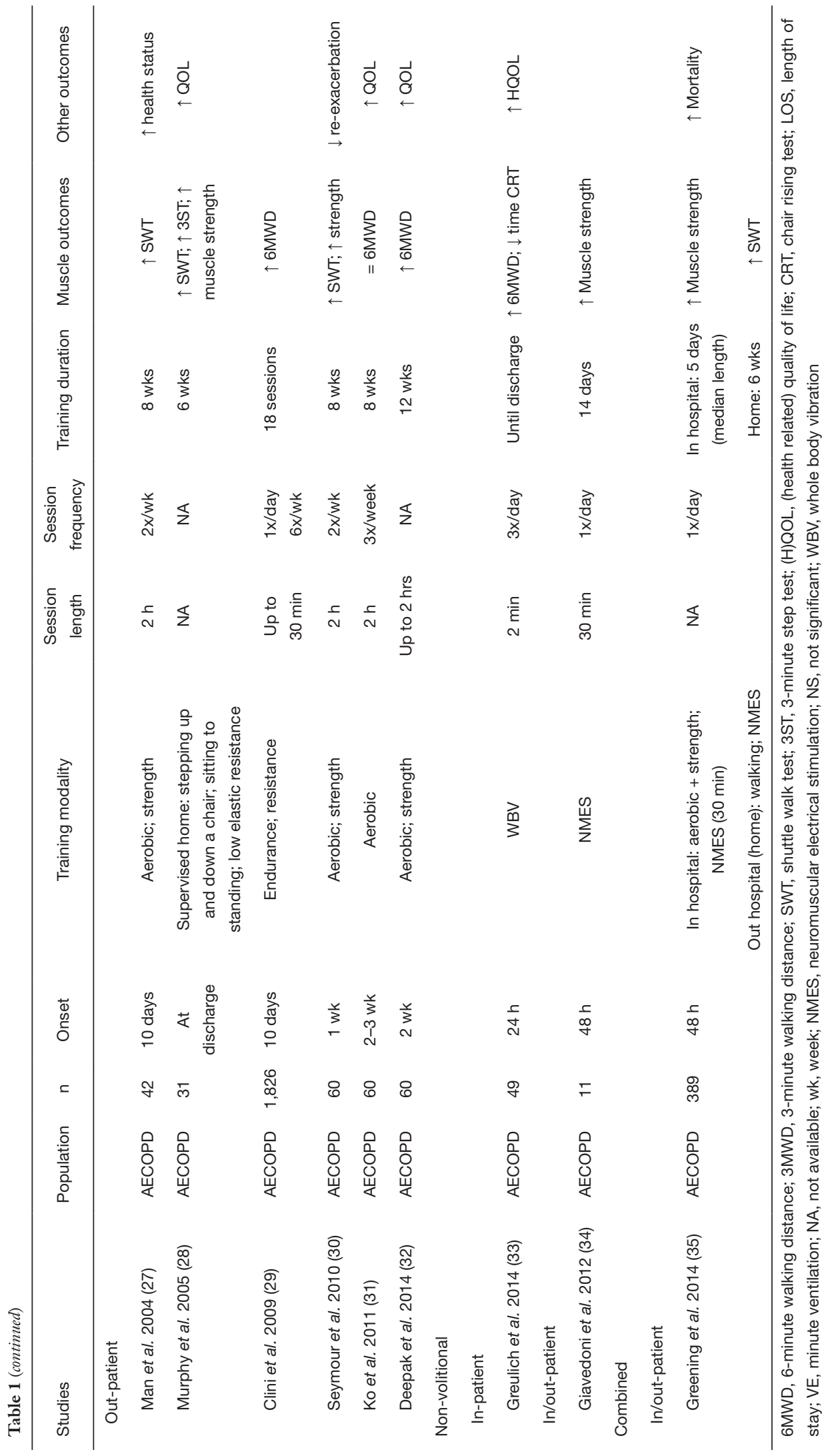


rehabilitation. The study of Kirsten et al. (15) shows that in that case an in-hospital short-term exercise program initiated 6-8 days after admission when patients had improved in order to be able to participate in the protocol significantly improved exercise capacity after 10 days as shown by the enhanced walking distance and minute ventilation compared to control patients.

There are also not enough data yet to support which training modalities would be the most appropriate but preliminary data from a small sample size trial underlined that an in-patient low-intensity exercise program based on aerobic and resistance training might be preferable to gain the most benefit for functional outcomes such as walk test, muscle training and Barthel index (17). Similarly, a shortterm exercise intervention using a pedal exerciser during hospital stay suggested that this type of exercise program focusing on lower limbs was efficient to reduce disability in frail elderly patients with acute exacerbation of COPD (22). At hospital discharge, muscle strength, balance and exercise capacity were all improved compared to the control group not receiving pulmonary rehabilitation. This suggests that exercise training is likely to be feasible even in fragile population while the modalities of the intervention are adapted accordingly. More complete and comprehensive respiratory rehabilitation specific for acute exacerbation has been recently addressed (20). Thus, a respiratory rehabilitation exercise training package including six components has been developed for in-patients with acute exacerbation of COPD. The six components included disease awareness, sputum clearance treatment, purse-lip breathing training, upper-limb exercise with deep training, walk training, and assigned pulmonary rehabilitation coordinators (to assist patients in nutrition management and health education). This 4-day intervention reduced symptoms such as dyspnea and cough and increased exercise tolerance in hospitalized elderly patients with acute exacerbation of COPD (20). The relevance of using such comprehensive respiratory rehabilitation exercise training package deserves further studies.

Taking together, it seems that the short-term duration of the inpatient pulmonary rehabilitation is feasible and may lead to improvement in several outcomes such as functionality, symptoms, health related quality of life and eventually reduced hospital readmission but it is unlikely to be associated with noticeable beneficial effects on activity of daily living. However, intuitively, we expect inpatient rehabilitation to be essential for recovery after exacerbation. This strategy is rather aimed at limiting or preventing the consequences of exacerbation and hospitalization on muscle function such as loss of muscle strength, reduced exercise capacity and physical activity, and health-related quality of life. Obviously, such intervention would benefit from further rehabilitation after discharge either as inhospital training or home-based rehabilitation or at least by encouraging the patients to do some simple home exercises. A recent case study, however, suggests that the referral rate of COPD patients after hospital discharge to participate in early rehabilitation is extremely low if not actively stimulated and remains insufficient anyway (36).

\section{Inpatient-outpatient program}

Studies examining the impact of a rehabilitation program started within days after admission and continued for weeks or months after discharge are scarce and differ in term of training modalities as detailed thereafter.

Whether early pulmonary rehabilitation would reduce acute health-care utilization was addressed in elderly COPD patients hospitalized for an acute exacerbation with severe impairment of pulmonary function, poor health related quality of life and high COPD-related morbidity (25). In this prospective randomized controlled study, an in-patient program was initiated at a mean of 2.6 days after admission and consisted of walking and limb strength exercise (30 min a day). It was followed by a hospital-based outpatient program consisting in $1 \mathrm{~h} /$ session of supervised exercise training, twice weekly, for 8 weeks. Moreover, at the end of this outpatient program, patients were guided to continue with a 30-min daily activity program established from their supervised exercise program. This intervention resulted in a trend to a greater improvement of exercise capacity index at 3 months compared to patients receiving usual care (25). A trend for reduced COPD-readmissions, timeto-readmissions and length of hospital stays was also observed (25). In this study, several efforts were also made to help the patient attending the rehabilitation program after discharge or to motivate them to be engaged in some active exercise. For that purpose, free transport was provided and they also received a government-funded opportunity to attend their local community gym. It is probably wise to add such facilitating strategies to guarantee a better compliance of the rehabilitation after discharge in particular transport facilities may not be evident for this frail population especially for those living in a rural zone.

Whether a home-based exercise program could maintain the benefits of a short-term hospital-based exercise program was examined in a randomized control 
study including severe COPD patients hospitalized for an acute exacerbation (23). A 10-day walking training program was started 4-7 days after admission when the patients' condition had stabilized and was followed by a 6-month home based walking program integrated in daily life activities. A significant improvement in the $6 \mathrm{MWD}$ was observed in the training group after 10 days and this improvement was maintained over 6 months (23) and up to 18 months (24). Quality of life was also improved in the training group during hospitalization and this effect was maintained over time $(23,24)$. In addition, disease-related medical consumption such as number of hospital admission and use of medication was significantly less after 18 months in the training group (24).

These data based on pilot studies are encouraging as they suggest that long-term beneficial effects of pulmonary rehabilitation involving active training might be expected when an in- and outpatient program is delivered to COPD patients with acute exacerbation even in severe and frail patients. However, more studies are warranted to confirm these data.

\section{Out-patient program}

Several studies have focused on hastening recovery after hospitalization following an acute exacerbation and have investigated the potential of short-term pulmonary rehabilitation program in outpatients. The time at which pulmonary rehabilitation was initiated varies between studies (either immediately after hospitalization or within days or weeks) as well as the duration and the modalities of the program. The pulmonary rehabilitation program is essentially based on active training and was usually delivered via a multidisciplinary team either in a rehabilitation center or as a supervised home-based program.

In a pilot study, a supervised home exercised program with simple exercises (stepping up and down a stair, sitting to standing from a chair, low-impact elastic resistance using Thera-Band) was initiated immediately after hospitalization for 6 weeks (28). This intervention resulted in improvement of exercise capacity and quality of life and increased muscle strength although the latter failed to reach statistical significance. At 3-month follow-up, there was a reduction in subsequent exacerbations of COPD for the exercised group. This study seems to suggest that a homebased training initiated shortly after an exacerbation for a relatively short period may help improving recovery after a COPD exacerbation. In addition, this study underlines that this early intervention was safe and well-tolerated.
Similarly, when the pulmonary rehabilitation program (aerobic and strength training) was started within 10 days after hospital discharge for 8 weeks, significant improvements in exercise capacity and health status were observed at three months (27). This was confirmed in a subsequent study of the same group where rehabilitation following the same modalities was associated with a reduced frequency of re-exacerbation requiring hospitalization in the subsequent 3 months (30). Quadriceps muscle strength was also enhanced after rehabilitation and this was correlated with change in walking performance (30). The study of Deepak et al. (32) further confirmed that early pulmonary rehabilitation (limb strengthening and aerobic activities) post exacerbation for 12 weeks had significant benefits on quality of life and exercise capacity.

In agreement with those small sample size studies, a large observational retrospective study where a rehabilitation program consisting in mixed physical training based on endurance and resistance of the peripheral muscle applied within 10 days after acute exacerbation indicated the feasibility of early inpatient rehabilitation in a population with severe deconditioning and unstable state (29). Moreover, rehabilitation, independently of MRC grade, resulted in clinically relevant improvement in $6 \mathrm{MWD}$ especially in patients who presented with the highest degree of disability.

Even when the pulmonary rehabilitation program was initiated later after hospital discharge (2-3 weeks), improvement in quality of life was observed up to 6 months although this intervention did not reduce health-care utilization at one year (31).

The time at which pulmonary rehabilitation should be initiated after hospital is still not known. Actually only one study have addressed the effects of early versus late pulmonary rehabilitation in COPD patients with exacerbation but solely on exacerbation rates, health related quality of life and mortality (26). In this multicenter randomized controlled trial, a 12 -week pulmonary rehabilitation program consisting in endurance and strength training and calisthenics was performed either within 2 weeks (early) or 6 months (late) after randomization. Although the timing of pulmonary rehabilitation did not affect exacerbation rates, a trend for greater benefits in terms of symptoms and health related quality of life was observed over the entire 18-month follow-up period in the early rehabilitation group. These data suggest that early rehabilitation postexacerbation may accelerate recovery from an exacerbation. 
All together, these data suggest that even when rehabilitation was initiated after hospital discharge following an acute exacerbation and although the training modalities differed between studies, this intervention was still able to result in several improvements in muscle function eventually associated with a reduced frequency of re-exacerbation requiring hospitalization.

\section{Non-volitional training (Table 1)}

As mentioned earlier, in non-volitional training, muscle contraction is not induced voluntarily by the patient but is induced by the intervention. There are currently two types of non-volitional training that have been delivered to COPD patients with acute exacerbation. The most popular is neuromuscular electrical stimulation (NMES) and the other one that has been recently introduced as a novel exercise training modality is whole body vibration (WBV).

With NMES, electrical stimulation is applied to limb muscles via electrodes placed on the skin and this induces muscle contractions. Passive training with NMES has been used as an alternative strategy to increase muscle performance especially in respiratory compromised patients unable or not willing to perform active training as this technique does not require patient's cooperation. A great advantage of this technique is its limited impact on ventilatory requirements and dyspnea (37) and its lower metabolic response compared to that of resistance training (38). It can also improve muscle strength without increasing cardiac load (39). Because NMES of limb muscles is better tolerated than whole body exercise especially in COPD patients with acute exacerbation, it has therefore been preferentially used as training modality in these patients (40).

Whole body vibration consists in an external stimulation that induces oscillation vibration to a subject standing with both feet on a vibrating platform. The stretch to the muscles of the lower extremities caused by the rapid oscillating movement of the platform induced rapid muscle contractions through a neuromuscular reflex. Vibration can be transferred to both feet synchronously or using a sidealternating mode (41).

For the time being, there are only two pilot studies examining the potential of these non-volitional training modalities in COPD patients with acute exacerbation: one dealing with whole body vibration delivered as an in-patient program and the one with NMES based on an in-outpatient program.

A proof of concept study recently addressed the potential of WBV training in COPD patients hospitalized for an exacerbation (33). In this randomized control trial, the training program started the day of admission or the day after and consisted in either a whole-body vibration training in addition to conventional physiotherapy or to a physiotherapy program alone. This study highlighted that WBV was well tolerated by the exacerbated COPD patients, it did not promote systemic inflammation or cause adverse effects. This strategy resulted in significant improvement of exercise capacity and quality of life. This beneficial effect was further underlined by the enhanced serum levels of exercise and muscle activity markers such as PGC1- $\alpha$ and irisin. These data suggest that WBV may be a useful training modality to be added to conventional exercise training during hospitalization for acute exacerbation. However, these data need to be confirmed in larger studies where training modalities of WBV (e.g., optimal intensity and duration) should be addressed.

The feasibility and efficacy of NMES as early intervention to prevent muscle function deterioration due to severe exacerbation in COPD patients has been investigated in a pilot study (34). A daily session of NMES during 14 days starting within 48 hrs of hospital admission and continuing at home after discharge was applied to one leg. This shortterm intervention not only prevented the loss of quadriceps muscle force assessed as maximal voluntary contraction but it also improved strength in the stimulated leg compared to the control leg. This study also indicated that the NMES was well tolerated by the patients who were able to increase the training stimulation intensity throughout the treatment duration, it did not induce any adverse effects and importantly self-administration was possible (34).

Although these data are encouraging, they definitely need to be confirmed in larger clinical trials. In particular, the potential of these non-volitional techniques may be particularly attractive for acute setting situation and this merits to be better addressed in future studies.

\section{Combined active with non-volitional training (Table 1)}

Only one study has examined the combination of active training with a non-volitional training (35). This prospective randomized control trail included 389 hospitalized patients randomized to either usual care or to a 6-week intervention initiated within $48 \mathrm{hrs}$ of hospital admission. Daily supervised volitional (aerobic and strength training) and non-volitional (NMES) was delivered for the duration of hospitalization (median length: 5 days) with the exercise program being individually personalized and progressed. This in-patient program was followed at hospital discharge by a supported 
self-management and exercise program consisting in progressive walking-based home exercise and daily NMES, patients' diaries and telephone consultations. However, limited gains in terms of muscle strength improvement and field walking performance were reported three months after discharge (35). Interestingly, the endurance shuttle walk test was significantly higher in the early rehabilitation group but only at six weeks (35). Enhanced gain in performance was observed in the subgroup of patients who were not readmitted to hospital suggesting that the benefits of the intervention were maintained as long as it was not disrupted by unscheduled readmission to hospital (35). Unfortunately, the intervention did not reduce the number of readmissions in the subsequent 12 months but it increased the risk of death at 12 months. The latter finding is disturbing and could not be really explained by the results and the authors concluded that caution was warranted before implanting such program during the immediate recovery from acute illness.

\section{Meta-analysis}

The Cochrane review on randomized controlled trials on pulmonary rehabilitation following exacerbation of COPD has been recently updated (42). Eleven studies were added so that the update review included 20 studies (18 peer reviewed articles and two abstracts) with 1,477 participants. The majority of the individual randomized controlled trials described previously in the current review has been included in the update version of the Cochrane review though with some exceptions $(22,26)$. The meta-analysis showed that pulmonary rehabilitation is a safe intervention for COPD patients who had experienced an acute exacerbation. It did improve quality of life and exercise capacity while the effects on hospital readmissions and mortality remained unclear. Presence of heterogeneity regarding the data on hospital readmissions and mortality might be explained for a part by the extensiveness of the rehabilitation programs and by the methodological quality of the included studies. The authors recommended to further explore whether the effects of rehabilitation after COPD exacerbations are depending on the extent of the pulmonary rehabilitation program and its modalities of implantation (in/ out patient care, hospital- or home-based).

\section{Early rebabilitation in bospitalized patients with ventilatory support}

Despite optimal treatment, some patients with exacerbation may require ventilator support because of acute respiratory failure. In case invasive mechanical ventilation is used, time to extubation may be lengthen with some patients even requiring tracheotomy and prolong mechanical ventilation with the risk to become ventilator dependent. This may promote loss of muscle function and increased morbidity and mortality. Therefore, pulmonary rehabilitation has been recently introduced in ICU to determine its feasibility and the extent to which it could help improving muscle weakness among other things. In ICU, pulmonary rehabilitation includes strategies such as positioning, mobilization, exercise, electrical stimulation, respiratory muscle training, but also airway cleaning, noninvasive mechanical ventilation and removing from ventilation. There are currently very few studies examining the effects of pulmonary rehabilitation in ventilated COPD patients following exacerbation. Stepwise pulmonary rehabilitation program and NMES alone or combined with mobilization have been up to now investigated. All these studies refer to in-patient program.

\section{Comprehensive rehabilitation program (Table 2)}

The first prospective randomized controlled study addressing the efficacy of a rehabilitation program in COPD recovering from an episode of acute respiratory failure was performed about 20 years ago (43). Clinically stable patients were enrolled 3 to 5 days after admission to the respiratory intensive care unit. Two third of the patients was ventilated (62\% invasively in the rehabilitation group versus $69 \%$ in the standard therapy plus progressive deambulation group). Most patients under invasive mechanical ventilation were tracheotomized. The rehabilitation program consisted in a comprehensive stepwise program including passive mobilization (step I), early deambulation (step II), respiratory and lower skeletal muscle training (step III) and if feasible for the patients, a complete lower extremity training with treadmill (step IV). Actually most of the patients performed step III task while one third was enrolled into step IV. At discharge, 6MWD and maximal inspiratory pressure (MIP) as well as dyspnea were significantly improved in the rehabilitation group compared to patients under standard therapy and about $87 \%$ of the patients regained walking autonomy after the rehabilitation program. At that time, the author concluded that a comprehensive rehabilitation program should be started early in every COPD patient admitted to an intensive care environment.

\section{Non-volitional program (Table 2)}

Whether NMES was able to counterbalance the adverse 
Table 2 Studies on early rehabilitation in hospitalized COPD patients with ventilatory support

\begin{tabular}{|c|c|c|c|c|c|c|c|c|c|c|}
\hline In-patient & References & Population & $n$ & Onset & Training modality & $\begin{array}{l}\text { Session } \\
\text { length }\end{array}$ & $\begin{array}{l}\text { Session } \\
\text { frequency }\end{array}$ & $\begin{array}{l}\text { Training } \\
\text { duration }\end{array}$ & $\begin{array}{c}\text { Muscle } \\
\text { outcomes }\end{array}$ & $\begin{array}{c}\text { Other } \\
\text { outcomes }\end{array}$ \\
\hline Comprehensive & $\begin{array}{c}\text { Nava et al. } \\
1998(43)\end{array}$ & $\begin{array}{c}\text { Severe COPD; } \\
\text { 2/3 IMV }\end{array}$ & 80 & $\begin{array}{l}\text { 3-5 days } \\
\text { after } \\
\text { admission } \\
\text { to RICU }\end{array}$ & $\begin{array}{c}\text { Step I: passive } \\
\text { mobilization } \\
\text { Step II: early } \\
\text { deambulation } \\
\text { Step III: respiratory } \\
\text { and lower skeletal } \\
\text { muscle training } \\
\text { Step IV: lower } \\
\text { extremity training } \\
\text { with treadmill }\end{array}$ & $\begin{array}{c}30-45 \\
\min \end{array}$ & $2 x /$ day & $\begin{array}{c}\text { Until } \\
\text { discharge }\end{array}$ & $\uparrow 6 \mathrm{MWD} ; \uparrow \mathrm{MIP}$ & $\downarrow$ dyspnea \\
\hline \multirow[t]{2}{*}{ Non-volitional } & $\begin{array}{c}\text { Zanotti } \\
\text { et al. } 2003 \\
(44)\end{array}$ & $\begin{array}{c}\text { COPD; IMV } \\
\text { (tracheotomized); } \\
\text { bed bound }\end{array}$ & 24 & NA & $\begin{array}{c}\text { NMES + active } \\
\text { limb mobilization }\end{array}$ & $\begin{array}{l}\text { Up to } \\
30 \mathrm{~min}\end{array}$ & $5 x / w k$ & 4 weeks & $\begin{array}{c}\uparrow \text { muscle } \\
\text { strength; } \downarrow \text { time } \\
\text { bed to chair }\end{array}$ & - \\
\hline & $\begin{array}{l}\text { Abdellaoui } \\
\text { et al. } 2011 \\
\text { (45) }\end{array}$ & $\begin{array}{c}\text { Severe COPD; 1/3 } \\
\text { IMV (intubated); } \\
\text { 1/3 NIV }\end{array}$ & 15 & $\begin{array}{l}12 \pm 8 \\
\text { days after } \\
\text { admission } \\
\text { to RICU }\end{array}$ & $\begin{array}{c}\text { NMES + } \\
\text { active-passive } \\
\text { mobilization }\end{array}$ & $1 \mathrm{~h} /$ day & $5 x / w k$ & 6 weeks & $\begin{array}{c}\uparrow 6 \mathrm{MWD} ; \uparrow \\
\text { muscle strength; } \\
\uparrow \text { type I; } \downarrow \text { protein } \\
\text { carbonylation }\end{array}$ & \\
\hline Combined & $\begin{array}{l}\text { Akar et al. } \\
2017 \text { (39) }\end{array}$ & $\begin{array}{l}\text { Conscious COPD; } \\
\text { IMV (intubated) }\end{array}$ & 30 & $\begin{array}{l}\text { Earlier but } \\
\text { time not } \\
\text { specified }\end{array}$ & $\begin{array}{c}\text { NMES + active } \\
\text { exercise }\end{array}$ & NA & $5 x / w k$ & $\begin{array}{c}20 \\
\text { sessions }\end{array}$ & $\begin{array}{c}\uparrow \text { muscle } \\
\text { strength; } \downarrow \text { blood } \\
\text { cytokines }\end{array}$ & - \\
\hline
\end{tabular}

IMV, Invasive mechanical ventilation; NIV, non-invasive ventilation; RICU, respiratory intensive care unit; 6MWD, 6-minute walking distance; MIP, maximal inspiratory pressure; NA, not available; wk, week; NMES, neuromuscular electrical stimulation.

effect of exacerbation on muscle function was addressed in a pilot study conducted in severe COPD patients recovering from an acute exacerbation (45). At admission to the ICU, one third of the patients was under non-invasive ventilation and another third was intubated and submitted to invasive mechanical ventilation. All patients received daily an activepassive mobilization in addition to a sham (sham group) or an effective (NMES group) electrostimulation for the same duration. The NMES program was initiated at $12 \pm 8$ days of hospitalization in the respiratory ICU and consisted in $1 \mathrm{~h}$ session per day, 5 days per week for 6 weeks ( 30 sessions in total). The NMES program was associated with enhanced quadriceps and hamstring maximal voluntary contraction as well as 6-minute walking distance in comparison with the sham group. There was also an increased proportion of the type I muscle fiber (fatigue resistant), a reduction in protein carbonylation especially of the myosin heavy chain and no aggravation of the local oxidative stress (45). Patients completed $29 \pm 1$ sessions out of 30 and no adverse effects were observed. This pilot study suggests that the NMES program might be a helpful tool to facilitate muscle function recovery in COPD patients following an
ICU hospitalization for exacerbation. Interestingly, the setting of the NMES protocol was appropriate to result in improvement of muscle function without inducing oxidative stress within the muscle.

In bedridden COPD patients with muscle hypotonia and atrophy receiving invasive mechanical ventilation via a tracheotomy, active limb mobilization was compared to NMES combined with active limb mobilization (44). Sessions were delivered at the patient's bed, 5 days a week for 4 weeks with the duration of session being gradually increased up to $30 \mathrm{~min}$. Both interventions led to significantly improvement of limb muscle strength but this effect was significant more pronounced in the group submitted to NMES in addition to active limb mobilization. In addition, the time needed to move patients from bed to chair was shortened when NMES was combined with active limb immobilization. At the end of the training period, the degree of impairment ranged between $25-50 \%$ and the patients were able to move again their muscles against gravity. This is likely to result in improved functionality and eventually quality of life. This study underlined the safety and feasibility of using NMES in severe and bed bound 
COPD patients under invasive mechanical ventilation but it also emphasized that this intervention further improved muscle strength compared to active mobilization alone.

Along the same line, a recent study compared the efficacy of active exercise combined or not with NMES ( $5 \mathrm{~d} /$ week, 20 sessions) started in earlier period of invasive mechanical ventilation in conscious and intubated COPD patients experiencing an acute exacerbation (39). This study showed that NMES alone or combined with active extremity exercise training did prevent loss of muscle strength in ICU and did even improve lower extremity muscle function after acute exacerbation (39). It also reduced inflammatory cytokines in blood. However, in contrast to the study of Zanotti et al. (44), the intervention did not shorten the time needed to move from bed to chair. As for the previous study, these data need to be confirmed in a larger sample size population.

These pilot studies suggest that pulmonary rehabilitation in ventilated COPD patients is likely to be feasible and might be efficient in improving muscle function and this may eventually facilitate recovery after acute exacerbation. More studies are needed before firm conclusions can be drawn.

\section{Nutritional support}

\section{Rationale}

During acute exacerbations, weight loss and muscle wasting are accelerated especially when exacerbation requires hospitalization. Energy balance is disturbed during the first days of hospitalization because of decreased dietary intake and increased resting energy expenditure $(7,8)$. Rather than decreased appetite, dyspnea and fatigue are likely to account for the inability to eat more prior and during the first days of hospitalization (8). Elevated levels of the appetite regulating hormone leptin and of pro-inflammatory cytokines are also potential contributor to the reduced energy intake of COPD patients during exacerbation $(7,8)$. This may be further compromised by the use of non-invasive mechanical ventilation where inadequate oral intake worsens with the time spent on non-invasive ventilation (46). Importantly, the negative nitrogen balance indicative of muscle wasting seen in hospitalized COPD patients with an acute exacerbation $(9,47)$ is associated with poorer prognosis. Low BMI and weight loss are, in fact, known as being unfavorable outcome during exacerbation predicting the need for mechanical ventilation (48). In addition, hospitalized COPD patients underweighted at baseline or with weight loss during the 12-month followup period are at increased risk for being hospitalized for an acute exacerbation (49).

\section{Modalities}

Nutritional supplementation has been achieved by using energy- or protein enriched diet. For the time being, two studies have investigated the impact of oral nutritional support on skeletal muscle in hospitalized COPD patients with acute exacerbation $(9,50)$.

\section{Nutritional intervention}

In a randomized clinical trial where COPD patients with an acute exacerbation received either oral nutritional supplementation (leading to an additional $10 \mathrm{kcal} / \mathrm{kg} / \mathrm{day}$ ) or usual feeding during hospitalization, inspiratory and limb muscle strength did not improve after 2 weeks but general well-being score tended to increase with oral nutritional support (9). Moreover, the majority of the patients were in negative nitrogen balance indicative of the presence of muscle wasting and a negative correlation between corticosteroid intake and nitrogen balance was reported. Changes in grip strength were also negatively correlated to corticosteroid intake during hospitalization showing that loss of muscle function was observed in patients receiving the highest doses of corticosteroids (9). This study underlined the difficulty to improve muscle wasting in COPD with acute exacerbation especially when treated with corticosteroids. Actually, apart from high doses of systemic corticosteroids, several factors may contribute to the negative nitrogen balance in COPD patients with acute exacerbation, those factors include enhanced inflammatory response, low protein intake and immobility. To be efficient nutritional support modalities would need to overcome the effects of these factors but should also take into account which of protein synthesis reduction and/or enhanced protein catabolism needs to be tackled.

Protein supplementation to increase protein synthesis and thus limit catabolic state was addressed in a randomized, double blind, placebo-controlled trial focusing on underweight or weight losing COPD patients hospitalized for an acute exacerbation (50). It was speculated that patients with recent weight loss would benefit more from nutritional intervention than (under)weight stable patients. While oral nutritional supplementation did increase energy and protein 
intake during hospitalization in contrast to the normal hospital diet alone, this did not translate into improvement in fat free mass or muscle strength. Nonetheless, weight gain during hospitalization in the COPD patients with the recent body weight loss tended to be higher than in the weight stable patients. The duration of the nutritional intervention (mean hospital-stay 9 days) might have been too short to demonstrate true benefit on functional outcomes but at least dietary intake at discharge was significantly higher than habitual intake which is encouraging. Nevertheless, this study showed that the protein-rich and carbohydrate-rich supplement was well tolerated and the recommended protein intake could be reached. But efforts to improve nutritional status in these patients after discharge should be stimulated and home care should be considered especially during the first weeks after hospitalization.

In summary, data on nutritional support during acute exacerbation are limited and do not allow to drawn firm conclusions. However, these studies showed that oral supplementation was feasible although efficiency was not evident. Modalities of these interventions need to be optimized and should also consider including a nutritional strategy after discharge. Research on these issues is urgently needed.

\section{Conclusions}

Data in the literature support that pulmonary rehabilitation is a safe and effective strategy to improve exercise capacity and quality of life for hospitalized COPD with acute exacerbation. However, effects on hospital admissions and mortality are still heterogeneous and remain to be confirmed. The relevance of pulmonary rehabilitation for acute exacerbation is nevertheless, currently being recognized since recent international guidelines and statements have supported its delivery in acute setting for COPD with exacerbation $(51,52)$. Unfortunately, for patients under mechanical ventilation, there are not enough studies to establish the relevance of pulmonary rehabilitation in this setting although available data are encouraging. The same holds true for nutritional support that has been up to now poorly investigated although this intervention might be highly relevant especially in undernourished COPD patients particularly when exercising.

\section{Future perspectives}

Future research is still needed to refine pulmonary rehabilitation program for acute exacerbation. In particular, design and implementation of such program including the best timing to start with but also its continuity and setting in terms of best appropriate exercise protocol need to be determined. For non-ventilated patients, there are, however, evidences indicating that pulmonary rehabilitation are an effective intervention to improve health related quality of life and exercise capacity. Thus, in view of the devastating effect of exacerbation for COPD patients, there is currently an urgent need to establish strategic recommendations for pulmonary rehabilitation adapted to the acute setting of exacerbations and based on the available evidences. COPD patients experiencing an exacerbation should have the opportunity to benefit from a pulmonary rehabilitation program. In addition, strategies to motivate patients to participate to pulmonary rehabilitation need to be developed. Patient's experience and perception need to be taken into account as well as his/her preferences in terms of exercise modalities and settings. In addition, facilitating strategies (e.g., organized free transport) may also be helpful to guarantee patient uptake and adherence post-exacerbation. For ventilated patients, efforts are needed to stimulate research on pulmonary rehabilitation, there are actually several tools available and the potential of non-volitional training modalities should not be neglected. Finally, there is really a lack of trials investigating the potential of nutritional support in COPD during exacerbation, experts in this field should really design studies to address this highly relevant issue.

\section{Acknowledgements}

None.

\section{Footnote}

Conflicts of Interest: The author has no conflicts of interest to declare.

\section{References}

1. Wedzicha JA, Seemungal TA. COPD exacerbations: defining their cause and prevention. Lancet 2007;370:786-96.

2. Vogelmeier CF, Criner GJ, Martínez FJ, et al. Global strategy for the diagnosis, management, and prevention of chronic obstructive lung disease 2017 report: GOLD Executive Summary. Arch Bronconeumol 
2017;53:128-49.

3. Spruit MA, Gosselink R, Troosters T, et al. Muscle force during an acute exacerbation in hospitalised patients with COPD and its relationship with CXCL8 and IGF-I. Thorax 2003;58:752-6.

4. Pitta F, Troosters T, Probst VS, et al. Physical activity and hospitalization for exacerbation of COPD. Chest 2006;129:536-44.

5. Crul T, Spruit MA, Gayan-Ramirez G, et al. Markers of inflammation and disuse in vastus lateralis of chronic obstructive pulmonary disease patients. Eur J Clin Invest 2007;37:897-904.

6. Ehsan M, Khan R, Wakefield D, et al. A longitudinal study evaluating the effect of exacerbations on physical activity in patients with chronic obstructive pulmonary disease. Ann Am Thorac Soc 2013;10:559-64.

7. Creutzberg EC, Wouters EF, Vanderhoven-Augustin IM, et al. Disturbances in leptin metabolism are related to energy imbalance during acute exacerbations of chronic obstructive pulmonary disease. Am J Respir Crit Care Med 2000;162:1239-45.

8. Vermeeren MA, Schols AM, Wouters EF. Effects of an acute exacerbation on nutritional and metabolic profile of patients with COPD. Eur Respir J 1997;10:2264-9.

9. Saudny-Unterberger H, Martin JG, Gray-Donald K. Impact of nutritional support on functional status during an acute exacerbation of chronic obstructive pulmonary disease. Am J Respir Crit Care Med 1997;156:794-9.

10. Garcia-Aymerich J, Farrero E, Félez MA, et al. Risk factors of readmission to hospital for a COPD exacerbation: a prospective study. Thorax 2003;58:100-5.

11. Cote CG, Dordelly LJ, Celli BR. Impact of COPD exacerbations on patient-centered outcomes. Chest 2007;131:696-704.

12. Garcia-Aymerich J, Lange P, Benet M, et al. Regular physical activity reduces hospital admission and mortality in chronic obstructive pulmonary disease: a population based cohort study. Thorax 2006;61:772-8.

13. Seemungal TA, Donaldson GC, Paul EA, et al. Effect of exacerbation on quality of life in patients with chronic obstructive pulmonary disease. Am J Respir Crit Care Med 1998;157:1418-22.

14. Donaldson GC, Wilkinson TM, Hurst JR, et al. Exacerbations and time spent outdoors in chronic obstructive pulmonary disease. Am J Respir Crit Care Med 2005;171:446-52.

15. Kirsten DK, Taube C, Lehnigk B, et al. Exercise training improves recovery in patients with COPD after an acute exacerbation. Respir Med 1998;92:1191-8.

16. Troosters T, Probst VS, Crul T, et al. Resistance training prevents deterioration in quadriceps muscle function during acute exacerbations of chronic obstructive pulmonary disease. Am J Respir Crit Care Med 2010;181:1072-7.

17. Tang CY, Blackstock FC, Clarence M, et al. Early rehabilitation exercise program for inpatients during an acute exacerbation of chronic obstructive pulmonary disease: a randomized controlled trial. J Cardiopulm Rehabil Prev 2012;32:163-9.

18. Borges RC, Carvalho CR. Impact of resistance training in chronic obstructive pulmonary disease patients during periods of acute exacerbation. Arch Phys Med Rehabil 2014;95:1638-45.

19. He M, Yu S, Wang L, et al. Efficiency and safety of pulmonary rehabilitation in acute exacerbation of chronic obstructive pulmonary disease. Med Sci Monit 2015;21:806-12.

20. Liao LY, Chen KM, Chung WS, et al. Efficacy of a respiratory rehabilitation exercise training package in hospitalized elderly patients with acute exacerbation of COPD: a randomized control trial. Int J Chron Obstruct Pulmon Dis 2015;10:1703-9.

21. Matsui H, Jo T, Fushimi K, et al. Outcomes after early and delayed rehabilitation for exacerbation of chronic obstructive pulmonary disease: a nationwide retrospective cohort study in Japan. Respir Res 2017;18:68.

22. Torres-Sánchez I, Valenza MC, Cabrera-Martos I, et al. Effects of an exercise intervention in frail older patients with chronic obstructive pulmonary disease hospitalized due to an exacerbation: a randomized controlled trial. COPD 2017;14:37-42.

23. Behnke M, Taube C, Kirsten D, et al. Home-based exercise is capable of preserving hospital-based improvements in severe chronic obstructive pulmonary disease. Respir Med 2000;94:1184-91.

24. Behnke M, Jörres RA, Kirsten D, et al. Clinical benefits of a combined hospital and home-based exercise programme over 18 months in patients with severe COPD. Monaldi Arch Chest Dis 2003;59:44-51.

25. Eaton T, Young P, Fergusson W, et al. Does early pulmonary rehabilitation reduce acute healthcare utilization in COPD patients admitted with an exacerbation? A randomized controlled study. Respirology 2009;14:230-8.

26. Puhan MA, Spaar A, Frey M, et al. Early versus late pulmonary rehabilitation in chronic obstructive 
pulmonary disease patients with acute exacerbations: a randomized trial. Respiration 2012;83:499-506.

27. Man WD, Polkey MI, Donaldson N, et al. Community pulmonary rehabilitation after hospitalisation for acute exacerbations of chronic obstructive pulmonary disease: randomised controlled study. BMJ 2004;329:1209.

28. Murphy N, Bell C, Costello RW. Extending a home from hospital care programme for COPD exacerbations to include pulmonary rehabilitation. Respir Med 2005;99:1297-302.

29. Clini EM, Crisafulli E, Costi S, et al. Effects of early inpatient rehabilitation after acute exacerbation of COPD. Respir Med 2009;103:1526-31.

30. Seymour JM, Moore L, Jolley CJ, et al. Outpatient pulmonary rehabilitation following acute exacerbations of COPD. Thorax 2010;65:423-8.

31. Ko FW, Dai DL, Ngai J, et al. Effect of early pulmonary rehabilitation on health care utilization and health status in patients hospitalized with acute exacerbations of COPD. Respirology 2011;16:617-24.

32. Deepak TH, Mohapatra PR, Janmeja AK, et al. Outcome of pulmonary rehabilitation in patients after acute exacerbation of chronic obstructive pulmonary disease. Indian J Chest Dis Allied Sci 2014;56:7-12.

33. Greulich T, Nell C, Koepke J, et al. Benefits of whole body vibration training in patients hospitalised for COPD exacerbations - a randomized clinical trial. BMC Pulm Med 2014;14:60.

34. Giavedoni S, Deans A, McCaughey P, et al. Neuromuscular electrical stimulation prevents muscle function deterioration in exacerbated COPD: a pilot study. Respir Med 2012;106:1429-34.

35. Greening NJ, Williams JE, Hussain SF, et al. An early rehabilitation intervention to enhance recovery during hospital admission for an exacerbation of chronic respiratory disease: randomised controlled trial. BMJ 2014;349:g4315.

36. Morsø L, Jensen MS, von Plessen C, et al. Rehabilitation of Discharged Patients With Chronic Obstructive Pulmonary Disease-Are New Strategies Needed? Health Serv Res Manag Epidemiol 2017;4:2333392816687704.

37. Vivodtzev I, Pépin JL, Vottero G, et al. Improvement in quadriceps strength and dyspnea in daily tasks after 1 month of electrical stimulation in severely deconditioned and malnourished COPD. Chest 2006;129:1540-8.

38. Sillen MJ, Janssen PP, Akkermans MA, et al. The metabolic response during resistance training and neuromuscular electrical stimulation (NMES) in patients with COPD, a pilot study. Respir Med 2008;102:786-9.

39. Akar O, Günay E, Sarinc Ulasli S, et al. Efficacy of neuromuscular electrical stimulation in patients with COPD followed in intensive care unit. Clin Respir J 2017;11:743-50.

40. Sillen MJH, Speksnijder CM, Eterman RA, et al. Effects of neuromuscular electrical stimulation of muscles of ambulation in patients with chronic heart failure or COPD: a systematic review of the English-language literature. Chest 2009;136:44-61.

41. Gloeckl R, Heinzelmann I, Kenn K. Whole body vibration training in patients with COPD: A systematic review. Chron Respir Dis 2015;12:212-21.

42. Puhan MA, Gimeno-Santos E, Cates CJ, et al. Pulmonary rehabilitation following exacerbations of chronic obstructive pulmonary disease. Cochrane Database Syst Rev 2016;12:CD005305.

43. Zanotti E, Felicetti G, Maini M, et al. Peripheral muscle strength training in bed-bound patients with COPD receiving mechanical ventilation: effect of electrical stimulation. Chest 2003;124:292-6.

44. Thorsdottir I, Gunnarsdottir I. Energy intake must be increased among recently hospitalized patients with chronic obstructive pulmonary disease to improve nutritional status. J Am Diet Assoc 2002;102:247-9.

45. Reeves A, White H, Sosnowski K, et al. Energy and protein intakes of hospitalised patients with acute respiratory failure receiving non-invasive ventilation. Clin Nutr 2014;33:1068-73.

46. Abdellaoui A, Préfaut C, Gouzi F, et al. Skeletal muscle effects of electrostimulation after COPD exacerbation: a pilot study. Eur Respir J 2011;38:781-8.

47. Nava S. Rehabilitation of patients admitted to a respiratory intensive care unit. Arch Phys Med Rehabil 1998;79:849-54.

48. Vitacca M, Clini E, Porta R, et al. Acute exacerbations in patients with COPD: predictors of need for mechanical ventilation. Eur Respir J 1996;9:1487-93.

49. Hallin R, Koivisto-Hursti UK, Lindberg E, et al. Nutritional status, dietary energy intake and the risk of exacerbations in patients with chronic obstructive pulmonary disease (COPD). Respir Med 2006;100:561-7.

50. Vermeeren MA, Wouters EF, Geraerts-Keeris AJ, et al. Nutritional support in patients with chronic obstructive pulmonary disease during hospitalization for an acute exacerbation; a randomized controlled feasibility trial. Clin Nutr 2004;23:1184-92.

51. Spruit MA, Singh SJ, Garvey C, et al. An official 
American Thoracic Society/European Respiratory Society statement: key concepts and advances in pulmonary rehabilitation. Am J Respir Crit Care Med 2013;188:e13-64.

Cite this article as: Gayan-Ramirez G. Relevance of nutritional support and early rehabilitation in hospitalized patients with COPD. J Thorac Dis 2018;10(Suppl 12):S1400S1414. doi: $10.21037 /$ jtd.2018.03.167
52. Bolton CE, Bevan-Smith EF, Blakey JD, et al. British Thoracic Society guideline on pulmonary rehabilitation in adults. Thorax 2013;68 Suppl 2:ii1-30. 